\title{
An Overview of Swarm Robotics: Swarm Intelligence Applied to Multi-robotics
}

\author{
Belkacem Khaldi, Foudil Cherif \\ Department of Computer Science. \\ LESIA Laboratory, \\ University of Biskra, Algeria.
}

\begin{abstract}
As an emergent research area by which swarm intelligence is applied to multi-robot systems; swarm robotics (a very particular and peculiar sub-area of collective robotics) studies how to coordinate large groups of relatively simple robots through the use of local rules. It focuses on studying the design of large amount of relatively simple robots, their physical bodies and their controlling behaviors. Since its introduction in 2000, several successful experimentations had been realized, and till now more projects are under investigations. This paper seeks to give an overview of this domain research; for the aim to orientate the readers, especially those who are newly coming to this research field.
\end{abstract}

\section{General Terms}

Swarm robotics, swarm intelligence, multi-robot systems.

\section{Keywords}

Swarm robotics applications, swarm robotics simulators, swarm robotics problems classification.

\section{INTRODUCTION}

Inspired from the complex behaviors observed in natural swarm systems (e.g., social insects and order living animals), swarm intelligence (SI) is a new field that aims to build fully distributed de-centralized systems in which overall system functionality emerges from the interaction of individual agents with each other and with their environment. As a result to try applying the insight gained from this domain research into multi-robotics, an emerging research area called swarm robotics (SR) has been issued.

SR is the study of how to coordinate large groups of relatively simple robots through the use of local rules. It focuses on studying the design of large amount of relatively simple robots, their physical bodies and their controlling behaviors [1]. SR is closely related to the idea of SI and it shares its interest in self-organized decentralized systems. Hence, it offers several advantages for robotic applications such as scalability, and robustness due to redundancy [2].

This paper seeks to give an overview of SR for the aim to orientate the readers, especially those who are newly coming to this research field, the paper highlights the grand lines of the different main focuses areas in this domain research.

In the upcoming sections, we introduce in section 2 the SI as an emergent research domain inspired from nature swarms, followed by overviewing Multi-robot systems (MRS) in section 3. In section 4 we introduce $\mathrm{SR}$ as an application of SI technics to MRS. The remaining sections (section 5 to section 8) are for more details about SR, these sections involve: definition of SR and its features, its potential applications in real world, the classification of the problems being focused on, and finally exploring some real successful projects and simulations being realized in real experimentation.

\section{SWARM INTELLIGENCE}

Who among us haven't been amazed by the individually simple but collectively complex behavior exhibited by natural grouping systems including social insects such as: ant' colonies, termites, bees, wasps ...etc., and high order living animals such as: flocks of birds, fish schooling, and packs of wolves ...etc.? Inspired by the robustness, scalability, and distributed self-organization principles observed in these amazing natural collective complex behaviors emerged from individual simple local interactions rules, an attempt to apply the insight gained through this research to artificial systems (e.g., massively distributed computer systems and robotics) has given rise to a new research topic called (SI) [3]. This increasing domain research that is firstly introduced in the context of cellular robotic systems by Beni and Wang [4] is considered as a sub-field of artificial intelligence based around on the study of collective behaviors in de-centralized, selforganized systems [5]. Although there is no a specific definition for swarm intelligence, we adopt heir the one denoted by Dorigo \& Birattari [6]:

'The discipline that deals with natural and artificial systems composed of many individuals that coordinate using decentralized control and self-organization. In particular, the discipline focuses on the collective behaviors that result from the local interactions of the individuals with each other and with their environment'.

So, a swarm intelligence system consists typically of a population of relatively simple agents (relatively homogenous or there are a few types of them [6]) interacting only locally with themselves and with their environment, without having a global knowledge about their own state and of the state of the world. Moreover, the overall observed behavior is emerged in response to the local environment and to local interactions between the agents that follow often very simple rules [7].

Natural swarm based theories have been applied to solve analogous engineering problems in several domains engineering from combinatorial optimization to rooting communication network as well as robotics applications, etc. (for a recent comprehensive review, readers can refer to [8]). The most well-known swarm based algorithms are: Ant Colony Optimization Algorithms (ACO), Particle Swarm Optimization Algorithms (PSO), Artificial Fish Swarm Algorithm (AFSA) and Bee based Algorithms. The ACO algorithm is inspired from the foraging behavior of ant colonies in finding shortest paths from their nests to food sources. The source of inspiration of PSO based algorithms comes especially from the behavior observed in bird flocking or fish schooling when they are moving together for long 
distances to search for food sources, whereas The AFSO algorithm is inspired from the collective movement observed in the different behaviors exhibited by fishes such as searching for food, following other fishes, protecting the group against dangers and stochastic search [9]. Bee based algorithms can be classified into three different main groups: (1) the honeybee' foraging behavior based algorithms, (2) the ones based on mating behavior in honeybee, and (3) the queen bee evolution process based algorithms (more details can be find in [10].

\section{MULTI-ROBOT SYSTEMS (MRS)}

Multi-robot systems (MRS) are born to overcome the lack in information processing capability and many other aspects of single robots that are not capable to dial with special tasks; which, in order to be efficiently completed, need cooperation and collaboration between groups of robots [11]. Since its introduction in the late 1980s, various works (such as: cellular robotics, collective robotics, and distributed robotics) have been issued to describe group of simple physical robots collaborating together to perform specific tasks. MRS have also achieve a great success and made a great progress in many areas such as cooperative transportation and aggregation, environmental monitoring, search-and-rescue missions, foraging, and space exploration [12].

In such task; even the simplicity in design and the low-cost in productivity, as well as the increase in capabilities, flexibility, and fault tolerance advantages gained when using multi-robots instead of a single one; however with the new arising challenges such as decentralization in control and selforganization, researchers in multi robotic field begun to make attention to the increase progress known in swarm intelligence systems giving birth to the new sub-domain research "swarm robotics".

\section{SWARM ROBOTICS (SR)}

Swarm robotics is a very particular and peculiar sub-area of collective robotics in which swarm intelligence techniques are applied. The 2000-year has witnesses the first project "swarmbot" [13] that has been marked as the real period of the development of swarm robotics. Marco Dorigo, the inventor of ACO algorithm; shared this project for the aim to study new approaches to the design and implementation of selforganizing and self-assembling artifacts.

Marco Dorigo and Erol Sahin [13],[14] ones of the founders of swarm robotics gave a definition to this research domain as follow: 'Swarm robotics can be loosely defined as the study of how collectively intelligent behavior can emerge from local interactions of a large number of relatively simple physically embodied agents'. The main idea of the approach behind this domain research is to build relatively many small and lowcost robots that are supposed to accomplish the same task as a single complex robot or a small group of complex robots [15]. The approach also takes into account studying the design of robots (both their physical body and their controlling behaviors) in a way that a desired collective behavior emerges from the inter-robot interactions and the interactions of the robots with the environment [16].

Further; as the key properties (pointed out in [2]) of a typical SI system can be applied to ether MRS and SRs; a set of criteria has been highlighted by Dorigo and Shahine [17] to overcome the confusions raised about the use of the term "swarm" and the overlapping meanings applied to multi-robot research. Dorigo and Shahin' set criteria; which are not meant to be used as a checklist, rather they help evaluating the degree to which SR might be applied and how it might be different from other MRSs; are described as follow [12]:

- Autonomy: the swarm-robotic system is made up of autonomous robots that are able to physically interact with the environment and affect it.

- Large number: The swarm-robotic system should be consisted of limited homogeneous groups of robots in which each group contains of large number of members. Hence, highly heterogeneous robot groups tend to fall outside swarm robotics).

- Limited capabilities: the SR system is composed of robots relatively incapable or inefficient to carry out tasks on their own but they are highly efficient when they cooperate.

- Scalability and robustness: A swarm-robotic system should be scalable and robust. Increasing the number of unites will improve the performance of the overall system and on the other hand, reducing some units will not yield to a breakdown of the system.

- Distributed coordination: in SR, the coordination between robots is distributed; each robot should only have local and limited sensing and communication abilities.

Based on these criteria; SRS are more beneficent than MRS which might be used whenever several robotic platforms are applied to achieve a mission. The main benefits when using SR reside on [15]: (1) the robustness feature: explained by the coherency of the whole system when losing some robots; this can gain us money investment in hundreds of small swarm robots, rather than investing the same amount of money or greater in a single complex robot that can leads to the failure of the all over project if a single failure is persisted. (2) The flexibility feature: enlightened by rather needing a hardware reconfiguration of complex robots to accomplish a task, the same task is achieved by coordinated swarm robots that are not essentially personalized to a given task. (3) The scalability feature described by the fact that relying only on local information; a swarm robotic algorithm can be applied unchanged to a group of any (reasonable) size. The table below as it's deducted from [18] summarizes these critters of differentiation:

Table 1. Comparison of SR and MRS

\begin{tabular}{|lcc|}
\hline & Swarm robotics & MRS \\
\hline Population Size & Variation in great range & Small \\
\hline Control & $\begin{array}{c}\text { Decentralized and } \\
\text { autonomous }\end{array}$ & $\begin{array}{c}\text { Centralized/ } \\
\text { remote }\end{array}$ \\
\hline Homogeneity & Homogeneous & heterogeneous \\
\hline Flexibility & High & Low \\
\hline Scalability & High & Low \\
\hline Environment & Unknown & Known/unknown \\
\hline Motion & Yes & Yes \\
\hline
\end{tabular}

SI techniques as ACO and PSO can be used as a control algorithm for distributed robot swarms, but a good problemsolving system does not have to be biologically relevant. However, the remarkable success of social insects in surviving and colonizing our planet can serve as a starting point for new metaphors in engineering and computer science [16]. 


\section{POTENTIAL APPLICATION OF SWARM ROBOTICS}

Since the emergent of swarm robotics research field, several works have been issued to explain how we can benefit from the properties of swarm robotics systems that make them appealing in several potential application domains. Swarm robotics have been involved in many tasks [1] such as the ones demanding miniaturization, like distributed sensing tasks in micro-machinery or the human body; those demanding cheap designs, such as mining task or agricultural foraging task; those requiring large space and time cost, and are dangerous to the human being or the robots themselves, such as post-disaster relief, target searching, military applications, etc. Refers to Ying TAN and Zhong-yang ZHENG [1],[18], swarm robotics is mostly used in:

\subsection{Tasks covering large area:}

Swarm robotics can be applied in tasks that require a large region of space. Heir; the robots are specialized for large coverage tasks (e.g. surveillance, demining, and search and rescue) and they are distributed in an unstructured or large environment (e.g. underwater or extraterrestrial planetary exploration) in which no available infrastructure can be used to control the robots. In such tasks, robot swarms are wellmatched because they are able to: act autonomously without the need of any infrastructure or any form of external coordination, detect and monitor the dynamic change of the entire area, locate the source, move towards the area and take quick actions. Moreover the robots, in such urgent situation, can aggregate into a patch in order to block the source as a temporary solution.

\subsection{Tasks dangerous to robot:}

In several dangerous tasks such as mine rescue and recovery, robots may be irretrievable after the task is accomplished; thus, it's economically acceptable to use swarm robotics with simple and cheap individuals rather than using complex and expensive robots. Moreover it's reasonably tolerable to apply swarm robots that provide redundancy for dealing with such dangerous tasks.

\subsection{Tasks require scaling population and redundancy:}

Swarm robotics can be also applied in situations in which it is difficult or even impossible to estimate in advance the resources needed to accomplish tasks such as search and rescue, tracking, and cleaning. An example for this situation is: clearing oil leakage after tank accidents; heir at the beginning of the task the population of swarm is highly maintained when the oil leaks fast and it's gradually reduced when the leak source is plugged and the leaking area is almost cleared. The solution needed in these cases should be scalable and flexible; therefore a robot swarm could be an appealing solution: robots can be added or removed in time without any significant impact on the performance to provide the appropriate amount of resources and meet the requirements of the specific task. This can be respected by the robustness feature of swarm robotics that is the main benefits from redundancy of the swarm.

\section{SWARM ROBOTICS PROBLEMS FOCUS}

In the last decade, swarm robotics researches has known a significant progress due to the advantages gained when using such technology to solve many problems that are beyond the capabilities of classical multi-robots systems. The problems involves in swarm robotics research can be classified into [1]: those mainly based on the patterns (e.g. aggregation, cartography, migration, self-organizing grids, deployment of distributed agents and area coverage); those focused on the entities in the environment (e.g. Searching for the targets, detecting the odor sources, locating the ore veins in wild field, foraging, rescuing the victims in disaster areas and); and those mostly hybrid of the two previous problems (e.g. cooperative transportation, demining, exploring a planet and navigating in large area).

[19] Illustrates another classification of the problems involved in swarm robotics based on the collective behavior problems focus. In (Table 2) we summaries his study basing on giving: a short definition of the problem to be solved, its source of inspiration, the approaches used to model the problem, examples of the current researches that belongs to the problem, and finally the classification of the problem.

\section{INVOLVED PROJECTS AND SIMULATIONS}

\subsection{Swarm robotics involved projects}

From the emergent of swarm robotics as a novel research domain, several successful projects have been created in order to face the challenges raised in this area of research. The most but not list known projects are presented in (Table 3). The list is not exhaustive for all the available projects but it shows the most used swarm robots platforms.

\subsection{Swarm robotics simulation platforms:}

Using plenty of physical robots in swarm robotics researches is hardly difficult to afford; thus computer simulations are developed to visually test the structures and algorithms on computer before engaging in real physical robots tests. The use of computer simulations; which are generally easier to setup less expensive, normally faster and more convenient to use than physical swarms; is often very useful to perform prior to the investigation of real robots. In the section below we highlight the well-known widely used simulation platforms in swarm robotics researches.

\subsubsection{Player/stage}

Player/stage ${ }^{1}$ is a combined package of free Software tools for robot and sensor applications developed by the international team of robotics researchers under the GNU license.

Player component is one of the most widely used robot control interface in the world that provides a network interface to a variety of robot and sensor hardware. The control of robots can be programmed throw multi-programming language that can be run in any computer with a network connection to the robot. Stage component is a multiple robot simulator interfaced to Player, it simulates a population of mobile robots moving in and sensing a two-dimensional 2D bitmapped environment.

\subsubsection{Gazebo}

Gazebo $^{2}$ is a simulator that extends Stage for 3D outdoor environments. It includes an accurate simulation of rigid-body physics; hence the both realistic sensor feedback and possible interactions between objects can be then generated. Gazebo presents a standard Player interface in addition to its own

\footnotetext{
http://playerstage.sourceforge.net

${ }^{2}$ http://gazebosim.org/
} 
native interface. In this way, the controllers written for Stage can be used in Gazebo and vice-versa.

\subsubsection{UberSim}

The UberSim ${ }^{3}$ is a simulator developed at Carnegie Mellon for a rapid validation before loading the program to real robot soccer scenarios. UberSim uses ODE physics engine for realistic motions and interactions. Although originally designed for Soccer robots, the custom robots and sensors can be written in $\mathrm{C}$ in the simulator and the program can be uploaded to the robots using TCP/IP.

\subsubsection{USARSim}

USARSim $^{4}$, shorted for Unified System for Automation and Robot Simulation, is a high fidelity multi-robot simulator originally developed for search and rescue (SAR) research activities of the Robocup contest. It has now become one of the most complete general purpose tools for robotics research and education. It is built upon a widely used commercial game engine, Unreal Engine 2.0. The simulator takes full advantage

Table 2. Classifications of problems being studied in Swarm robotics

\begin{tabular}{|c|c|c|c|c|}
\hline Problem to be solved & Sources of inspiration & Modeling approaches & $\begin{array}{c}\text { Research literatures samples } \\
\text { (for more details refers to } \\
[19])\end{array}$ & $\begin{array}{c}\text { Problem } \\
\text { classification }\end{array}$ \\
\hline $\begin{array}{l}\text { Aggregation } \\
\text { - Clustering swarm robots in a } \\
\text { region of the environment. }\end{array}$ & $\begin{array}{l}\text { - Nature (e.g. Aggregation } \\
\text { bacteria, cockroaches, bees, fish } \\
\text { and penguins). }\end{array}$ & $\begin{array}{l}\text { - Probabilistic finite } \\
\text { state machines. } \\
\text { - Artificial evolution. }\end{array}$ & - [20] and [21] & \multirow{4}{*}{ 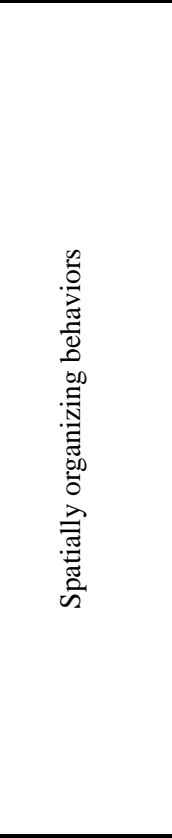 } \\
\hline $\begin{array}{l}\text { Pattern formation } \\
\text { - Deploying robots in a regular } \\
\text { and repetitive manner from } \\
\text { which specific distances are } \\
\text { kept between each other in } \\
\text { order to create a desired } \\
\text { pattern. }\end{array}$ & $\begin{array}{l}\text { - Biology (e.g. the spatial } \\
\text { disposition of bacterial colonies } \\
\text { and the chromatic patterns on } \\
\text { some animals). } \\
\text { - Physics (e.g. molecules } \\
\text { distribution and crystal } \\
\text { formation). }\end{array}$ & $\begin{array}{l}\text { - Virtual physics-based } \\
\text { design. }\end{array}$ & $-[22]$ & \\
\hline $\begin{array}{l}\text { Chain formation } \\
\text { - Auto-Positioning robots to } \\
\text { connect into two points. The } \\
\text { chain that they form can then } \\
\text { be used as a guide for } \\
\text { navigation or for } \\
\text { surveillance. }\end{array}$ & - Foraging ants. & $\begin{array}{l}\text { - Probabilistic finite } \\
\text { state machines. } \\
\text { - Virtual physics based } \\
\text { design. } \\
\text { - Artificial evolution. }\end{array}$ & $-[23]$ and [24] & \\
\hline $\begin{array}{l}\text { Self-assembly and } \\
\text { morphogenesis } \\
\text { - Connecting physically swarm } \\
\text { robots to each other to create } \\
\text { structures (morphologies). }\end{array}$ & - Ants (bridges, rafts, walls...). & $\begin{array}{l}\text { - Probabilistic finite } \\
\text { state machines. } \\
\text { - Artificial evolution. }\end{array}$ & $-[25]$ & \\
\hline Collective exploration & - Social animals (ants, bees...). & $\begin{array}{l}\text { - Probabilistic finite } \\
\text { state machines. } \\
\text { - Virtual physics-based } \\
\text { design. } \\
\text { - Network routing. }\end{array}$ & - [26] and [27] & \multirow{3}{*}{ 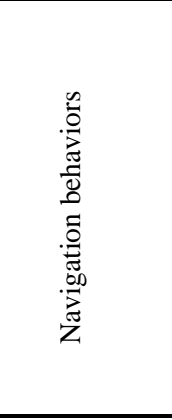 } \\
\hline $\begin{array}{l}\text { Coordination motion } \\
\text { - Moving in formation } \\
\text { similarly to schools of fish or } \\
\text { flocks of birds. } \\
\end{array}$ & $\begin{array}{l}\text { - Flocking in-group of birds. } \\
\text { - Schooling in group of fish. }\end{array}$ & $\begin{array}{l}\text { - Virtual physics-based } \\
\text { design. } \\
\text { - Artificial evolution. }\end{array}$ & $-[28]$ & \\
\hline $\begin{array}{l}\text { Collective transport } \\
\text { - Cooperating in order to } \\
\text { transport an object. }\end{array}$ & $\begin{array}{l}\text { - Cooperative carry prey in ant } \\
\text { colonies. }\end{array}$ & $\begin{array}{l}\text { - Probabilistic finite } \\
\text { state machines. } \\
\text { - Artificial evolution. } \\
\end{array}$ & $-[29]$ & \\
\hline $\begin{array}{l}\text { Consensus achievement } \\
\text { - Reaching consensus on one } \\
\text { choice among different } \\
\text { alternatives. }\end{array}$ & $\begin{array}{l}\text { - Ants' decision between the } \\
\text { shorter of two paths using } \\
\text { pheromones. } \\
\text { - Bees' decision between the best } \\
\text { foraging area and the best nest } \\
\text { location. } \\
\text { - Aggregation in Cockroaches. }\end{array}$ & $\begin{array}{l}\text { - Direct } \\
\text { communication. } \\
\text { - Indirect } \\
\text { communication. }\end{array}$ & - [30] and [31] & \multirow{2}{*}{ 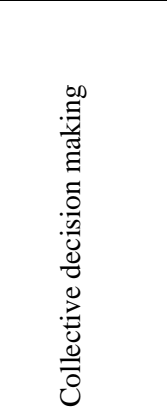 } \\
\hline $\begin{array}{l}\text { Task allocation } \\
\text { - Auto-distribution of swarm } \\
\text { robots over different tasks. } \\
\text { To maximize the } \\
\text { performance of the system. }\end{array}$ & $\begin{array}{l}\text { - Task allocation in ant and bee } \\
\text { colonies. }\end{array}$ & $\begin{array}{l}\text { - Probabilistic finite } \\
\text { state machines. }\end{array}$ & $-[32]$ & \\
\hline
\end{tabular}


of high accuracy physics, noise simulation and numerous geometrics and models from the engine. Evaluations have shown that USARSim can simulate the real time robots well enough for researchers due to the high fidelity physics engine.

\subsubsection{Enki}

Enki ${ }^{5}$ is an open source fast 2D physics based robot simulator written in $\mathrm{C}++$. It is able to simulate the robot swarms hundred times faster on the desktop computer than real-time robots. It is also able to simulate the kinematics, collision, sensors and cameras of robots working on a flat surface. Enki is built to support several existing real robot systems, including swarm-bots and E-puck, while user can customize their own robots into the platform.

Table 3. Some of successful Swarm Robotics Projects

\begin{tabular}{|c|c|c|}
\hline Project & Objective & Prototype \\
\hline $\begin{array}{c}\text { Open-source micro-robotic } \\
\text { Project } \\
\text { University of Stuttgart, Sergey } \\
\text { Kornienko and University of } \\
\text { Karlsruhe, Marc Szymanski, } \\
\text { Ramon Estane } \\
\text { http://www.swarmrobot.org }\end{array}$ & $\begin{array}{l}\text { Develop a cheap, reliable and swarm-capable micro-robot that can be } \\
\text { easily reproduced even at home. This robot allows building a large-scale } \\
\text { swarm system ( } 100 \text { and more robots) to investigate artificial self- } \\
\text { organization, emergent phenomena, and control in large robotic groups } \\
\text { and so on. This research is important to understand underlying principle } \\
\text { of information and knowledge processing, adaptation and learning for the } \\
\text { design and development of very limited autonomous systems. }\end{array}$ & $\begin{array}{l}\text { Cost: } £ 80 \\
\text { Sensor: distance, light, bearing } \\
\text { Motion/Speed: wheel, N/A } \\
\text { Size: } 3 \mathrm{~cm} \\
\text { Autonomy: } 1-2 \mathrm{~h}\end{array}$ \\
\hline $\begin{array}{c}\text { Swarm-bots project } \\
\text { IRIDIA, Université Libre de } \\
\text { Bruxelles } \\
\text { www.swarm-bots.org }\end{array}$ & $\begin{array}{l}\text { The project explores the design, implementation and simulation of self- } \\
\text { organizing and self-assembling artifacts. The project after it was } \\
\text { successfully completed in 2005; it has been extended by the swarmanoid } \\
\text { project, a project that proposes a highly innovative way to build } \\
\text { robots that can successfully and adaptively act in human made } \\
\text { environments. The swarm-bot prototype has been also used in e-swarm } \\
\text { project }\end{array}$ & $\begin{array}{l}\text { Cost: } N / A \\
\text { Sensor: range, bearing, camera, } \\
\text { bump, light } \\
\text { Motion/Speed: wheel, } 50 \mathrm{~cm} / \mathrm{s} \\
\text { Size: } 12.7 \mathrm{~cm} \\
\text { Autonomy: } 3 \mathrm{~h}\end{array}$ \\
\hline $\begin{array}{c}\text { E-puck education robot } \\
\text { École Polytechnique Fédérale De } \\
\text { Lausanne EPFL } \\
\text { http://www.e-puck.org }\end{array}$ & $\begin{array}{l}\text { The project develops a miniature mobile robot for education use. The } \\
\text { robots have several features specialized for such purpose. The robots } \\
\text { have a clean mechanical structure simple to understand, operate and } \\
\text { maintain. The robots are cheap and flexible, and can cover a large } \\
\text { spectrum of educational activities thanks to a large potential in sensors, } \\
\text { processing power and extension }\end{array}$ & $\begin{array}{l}\text { Cost: } £ 580 \\
\text { Sensor: distance, camera, } \\
\text { bearing, accelerometer, mic } \\
\text { Motion/Speed: wheel, } 13 \mathrm{~cm} / \mathrm{s} \\
\text { Size: } 7.5 \mathrm{~cm} \\
\text { Autonomy: } 1-10 \mathrm{~h}\end{array}$ \\
\hline $\begin{array}{c}\text { R-one project } \\
\text { Multi-Robot Systems Lab, Rice } \\
\text { University } \\
\text { http://mrsl.rice.edu/projects/r-one }\end{array}$ & $\begin{array}{l}\text { The project aims to provide an advanced low-cost mobile robots designed } \\
\text { for research, teaching and outreach, the developed robots was } \\
\text { successfully implicated in several projects such as multi robot } \\
\text { manipulation, distributed approach for exploring and triangulating an } \\
\text { unknown region, and distributed boundary detection. }\end{array}$ & $\begin{array}{l}\text { Cost: } N / A \\
\text { Sensor: distance, light, bump, } \\
\text { accelerometer, IR, localization } \\
\text { Motion/Speed: } \text { wheel, } 25 \mathrm{~cm} / \mathrm{s} \\
\text { Size: } 11 \mathrm{~cm} \\
\text { Autonomy: } 4 \mathrm{~h}\end{array}$ \\
\hline $\begin{array}{c}\text { Kilobot project } \\
\text { School of Engineering and } \\
\text { Applied Sciences } \\
\text { Wyss Institute for Biologically } \\
\text { Inspired Engineering } \\
\text { Harvard University } \\
\text { http://www.eecs.harvard.edu/ssr/ } \\
\text { projects/progSA/kilobot.html }\end{array}$ & $\begin{array}{l}\text { The project aims to design a robot system for testing the collective } \\
\text { algorithms with a population of hundreds or thousands of robots. Each } \\
\text { robot is made of low-cost parts and takes } 5 \text { min to be fully assembled. } \\
\text { The system also provides several overall operations for a large swarm, } \\
\text { such as updating programs, powering on, charging all robots and } \\
\text { returning home }\end{array}$ & $\begin{array}{l}\text { Cost: } £ 12 \\
\text { Sensor: distance, light } \\
\text { Motion/Speed: vibration, } 1 \mathrm{~cm} / \mathrm{s} \\
\text { Size: } 3.3 \mathrm{~cm} \\
\text { Autonomy: } 3-24 \mathrm{~h}\end{array}$ \\
\hline $\begin{array}{c}\text { Khepera III robot } \\
\text { K-Team Corporation } \\
\text { http://www.k-team.com/mobile- } \\
\text { robotics-products/khepera-iii }\end{array}$ & $\begin{array}{l}\text { Produced by K-Team corporation, the robot provides a new standard tool } \\
\text { for robotic experiments and demonstrations such as: artificial } \\
\text { intelligence, navigation, multi-Agents System, real-time programming, } \\
\text { control collective behavior, and advanced electronics demonstration. }\end{array}$ & $\begin{array}{l}\text { Khepera III } \\
\text { Cost: } / \text { /A } \\
\text { Sensor: range, bearing, camera, } \\
\text { bump, IR, ultrasonic, light } \\
\text { Motion } / \text { Speed: wheel, } 50 \mathrm{~cm} / \mathrm{s} \\
\text { Size: } 13 x 7 \mathrm{~cm} \\
\text { Autonomy: } 8 \mathrm{~h}\end{array}$ \\
\hline $\begin{array}{l}\text { Colias robot } \\
\text { Computational Intelligence Lab } \\
\text { (CIL), School of Computer } \\
\text { Science, University of Lincoln } \\
\text { http://colias.uk/index.htm }\end{array}$ & $\begin{array}{l}\text { The robot developed in this project has been designed as a complete } \\
\text { platform with supporting software development tools for robotics } \\
\text { education and research. The robot is a low-cost, open-platform, } \\
\text { autonomous micro-robot for robotic swarm applications. It has been } \\
\text { tested in both individual and swarm scenarios, and the observed results } \\
\text { demonstrate its feasibility for use as a micro-sized mobile robot and as a } \\
\text { low-cost platform for robot swarm applications. }\end{array}$ & $\begin{array}{l}\text { Cost: } £ 25 \\
\text { Sensor: distance, light, bump, } \\
\text { bearing, range } \\
\text { Motion/Speed: wheel, } 35 \mathrm{~cm} / \mathrm{s} \\
\text { Size: } 4 \mathrm{~cm} \\
\text { Autonomy: } 1-3\end{array}$ \\
\hline
\end{tabular}

\footnotetext{
${ }^{5}$ http://home.gna.org/enki/
} 


\subsubsection{Webots}

Webots $^{6}$ is a development environment used to model, program and simulate the mobile robots available for more than 10 years. With Webots, the user can design the complex robotic setups, with one or several, similar or different robots with a large choice of pre-defined sensors and actuators. The objects in the environment can be customized by the user. Webots also provides a remote controller for testing the real robots. Until now, Webots robot simulator has been used in more than 1018 universities and research centers in the worldwide.

\subsubsection{Breve}

Breve $^{7}$ is a free, open-source software package, which makes it easy to build 3D simulations of multi-agent systems and artificial life. Behaviors and interactions of agents are defined using Python. Breve uses ODE physics engine and OpenGL library that allows the observers to view the simulation in the 3D world from any position and direction. Users can interact at run time with the simulation using a web interface. Multiple simulations can interact and exchange individuals over the network.

\subsection{8 $V$-REP}

$\mathrm{V}$ - $\mathrm{REP}^{8}$ is an open source $3 \mathrm{D}$ robot simulator that allows creating entire robotic systems, simulating and interacting with dedicated hardware. V-REP is based on distributed control architecture: each object/model can be individually controlled via an embedded script, a plugin, a remote API client, or a custom solution. In V-REP, Controllers can be written in $\mathrm{C} / \mathrm{C}++$, Python, Java, Lua, Matlab, Octave or Urbi and can be directly attached to the objects in the scene and run simultaneously in both threaded and non-threaded fashions. This makes it very versatile and ideal for multi-robot application. V-REP is used for fast algorithm development, factory automation simulations, fast prototyping and verification, robotics related education, remote monitoring, safety double-checking, etc.

\subsubsection{ARGoS}

$\mathrm{ARGoS}^{9}$ was the official simulator of the Swarmanoid project; it is currently the main robot simulation tool for many European projects. ARGoS is a new pluggable, multi-physics engine for simulating the massive heterogeneous swarm robotics in real time. Contrary to other simulators, every entity in ARGoS is described as a plug-in one and easy to implement and use. In this way, the multiple physics engines can be used in one experiment, and the robots can migrate from one to another in a transparent way. Results have shown that ARGoS can simulate about 10000 wheeled robots with full dynamics in real-time. ARGoS is also able to be implemented in parallel in the simulation.

\subsubsection{TeamBots}

TeamBots $^{10}$ is a collection of Java applications and java packages for multi-agent mobile robotics research. The simulation environment is fully based on java, however some execution on mobile robots sometimes requires low-level libraries in C. It supports the prototyping, simulation and

\footnotetext{
${ }^{6}$ http://www.cyberbotics.com/

${ }^{7}$ www.spiderland.org/breve/

${ }^{8}$ http://www.coppeliarobotics.com/

${ }^{9} \mathrm{http} / / /$ iridia.ulb.ac.be/argos/

${ }^{10}$ www.teambots.org
}

execution of multi-robot control systems; which might be run either in simulation using the TBSim simulation application, or on mobile robots using the TBHard robot execution environment.

\subsubsection{MORSE}

MORSE$^{11}$ is a Blender Game Engine based simulator designed to provide a realistic 3D simulation of small to large environments, indoor or outdoor, with the ability to simulate one to tenths of autonomous robots. It comes with a set of robots base model (such as quadrotors, ATRV, Pioneer3DX, generic 4 wheel vehicle, PR2,...), with the possibility to add new ones.

\section{SUMMARY}

Swarm robotics is a relatively new research area that takes its inspiration from swarm intelligence and robotics. It is the result of applying swarm intelligence technics into multirobotics. Although a number of researches have been proposed, it's still quite far for practical application. In the present paper, an overview of swarm robotics has been given for better understanding of this multi-robot domain research and for clarifying the grand lines being focused on this domain. Interests that are newly coming to this topic research can be easily guided throw the different sections presented in this paper.

\section{REFERENCES}

[1] Ying T. 2013. Swarm Robotics: Collective Behavior Inspired by Nature. J Comput Sci Syst Biol. (2013), DOI= http://dx.doi.org/10.4172/jcsb.1000e106.

[2] Amanda J.C.S. 2007. Swarm Robotics and minimalism. Connection Science. 19(3), 2017. 245-260.

[3] William A. 2003. Modeling Artificial, Mobile Swarm Systems. Doctoral Thesis. Institute of Technology, California.

[4] Beni G., Wang J. 1989. Swarm Intelligence in Cellular Robotic. In Systems Proceedings of NATO Advanced Workshop on Robots and Biological Systems. 102(1989).

[5] Bighnaraj N., Sarita M., Subhra S., Swadhin K.B. 2012. Cooperative Swarm based Evolutionary Approach to find optimal cluster centroids in Cluster Analysis, IJCSI International Journal of Computer Science Issues. 9(2012)

[6] Dorigo M., Birattari M. 2007. Swarm Intelligence. Scholarpedia. 2(9), 2007.

[7] Eliseo F. 2009. A Control Architecture for a Heterogeneous Swarm of Robots, Rapport d'avancement de recherché $(\mathrm{PhD})$, Universite Libre De Bruxelles; Computers and Decision Engineering, IRIDIA.

[8] Rafael P., Heitor S. L. 2012. Theory and New Applications of Swarm Intelligence. InTech. Janeza Trdine 9, 51000 Rijeka, Croatia. ISBN 978-953-51-03646. 2012 .

[9] Mehdi N., Ghodrat S., Mehdi S., Adel N. T. 2012. Artificial fish swarm algorithm: a survey of the state of the-art, hybridization, combinatorial and indicative

\footnotetext{
${ }^{11}$ https://www.openrobots.org/wiki/morse/
} 
applications. Artif Intell Rev. Springer Science and Business Media B.V. 2012. Doi= 10.1007/s10462-0129342-2.

[10] Hamdi A., Monmarché N., Adel Alimi M., Slimane M. 2011. Bee-based algorithms: a review. http://www.researchgate.net/publication/236341293_Bee based_algorithms_a_review/file/72e7e51bea05a01b3c.pd f.

[11] Zhiguo S., Jun T., Qiao Z., Lei L., Junming W. 2012. A Survey of Swarm Robotics System. Advances in Swarm Intelligence Lecture Notes in Computer Science. 7331(2012).

[12] Hui Keng L. 2012. Error Detection in Swarm Robotics:A Focus on Adaptivity to Dynamic Environments. PhD Thesis. University of York, Department of Computer Science.

[13] Marco D., al. 2005. The SWARM-BOT Project. Swarm Robotics Lecture Notes in Computer Science. 3342(2005).

[14] Marco D., al. 2004. Evolving Self-Organizing Behaviors for a Swarm-Bot. Autonomous Robots. 17(2-3). September 2004. 223-245.

[15] Zur E. 2008. Space-Time Continuous Models of Swarm Robotic Systems: Supporting Global-to-Local Programming. PhD Thesis. Von der Fakultat fur Informatik der Universitat Fridericiana zu Karlsruhe $(\mathrm{TH})$

[16] Aleksandar J., Diego A. 2007. Swarm Intelligence and Its Applications in Swarm Robotics. 6th WSEAS Int. Conference on Computational Intelligence. ManMachine Systems and Cybernetics. Tenerife, Spain. December 14-16, 2007.

[17] Dorigo M., Sahin E. Guest editorial. 2004. Swarm robotics. Autonomous Robots. 17, 2-3(2004). 111-113.

[18] Ying T., Zhong-yang Z. 2013. Research Advance in Swarm Robotics. ScienceDirect. Defence Technology 9(2013). doi= http://dx.doi.org/10.1016/j.dt.2013.03.001.

[19] Manuele B., Eliseo F., Mauro B., Marco D. 2013. Swarm robotics: a review from the swarm engineering perspective. Swarm Intell. 7:1(2013). Doi=10.1007/s11721-012-0075-2.

[20] Garnier S., Jost C., Jeanson R., Gautrais J., Asadpour M., Caprari G., Theraulaz G. 2005. Aggregation behavior as a source of collective decision in a group of cockroachlike robots. In Lecture notes in artificial intelligence. Advances in artificial life. 3630(2005). 169-178.
[21] Soysal O., Bahçeci, E., Sahin E. Aggregations in swarm robotic systems: evolution and probabilistic control. Turkish Journal of Electrical Engineering and Computer Sciences, 15(2), 199-225.

[22] Shucker B., Bennett, J. K. 2007. Scalable control of distributed robotic macrosensors. In Distributed autonomous robotic systems. 6(2007). 379-388.

[23] Nouyan S., Campo A., Dorigo, M. 2008. Path formation in a robot swarm: self-organized strategies to find your way home. Swarm Intelligence, 2:1(2008). 1-23.

[24] Maxim P. M., Spears W. M., Spears D. F. 2009. Robotic chain formations. In Proceedings of the IFAC. workshop on networked robotic (NetRob'09).19-24

[25] O'Grady R., Christensen A., Dorigo M. 2009. SWARMORPH: multi-robot morphogenesis using directional self-assembly. IEEE Transactions on Robotics, 25(3), 738-743.

[26] Stirling T., Floreano D. 2010. Energy efficient swarm deployment for search in unknown environments. In Lecture notes in computer science. Proceedings of the 7 th international conference on swarm intelligence. 562563.

[27] Ducatelle F., Di Caro C. P. G. A., Gambardella L. M. 2011. Self-organized cooperation between robotic swarms. Swarm Intelligence. 5:2. 73-96.

[28] Turgut A. E., Çelikkanat H., Gökçe F., Sahin E. 2008. Self-organized flocking in mobile robot swarms. Swarm Intelligence. 2:2-4. 97-120.

[29] Groß R., Dorigo M. 2008. "Evolution of solitary and group transport behaviors for autonomous robots capable of self-assembling. Adaptive Behavior. 16:5. 285-305.

[30] Garnier S., Jost C., Jeanson R., Gautrais J., Asadpour M., Caprari G., Theraulaz G.2005. Aggregation behaviour as a source of collective decision in a group of cockroach-like robots. In Lecture notes in artificial intelligence. Advances in artificial life. 3630(2005). 169 178.

[31] Gutiérrez Á., Campo A., Monasterio-Huelin F., Magdalena L., Dorigo M. 2010. Collective decisionmaking based on social odometry. Neural Computing \& Applications. 19(6). 807-823.

[32] Pini G. 2014. Task partitioning in swarms of robots an adaptive method for strategy selection. URL http://iridia.ulb.ac.be/supp/IridiaSupp2011003/index.html. 